\title{
Ständiger Ausbau der Massnahmen zur Patientensicherheit
}

\author{
Isabelle Praplan \\ H+ Die Spitäler der Schweiz, Projektleiterin Qualität und Patientensicherheit (Geschäftsbereich Betriebswirtschaft)
}

Spitäler und Kliniken sind bestrebt, die bestmögliche Qualität zu erbringen und über die gesetzlichen Anforderungen hinauszugehen. Sie beteiligen sich über den Messplan des ANQ hinaus an freiwilligen Initiativen, führen Qualitätsmessungen durch, beteiligen sich an Registern und lassen sich zertifizieren. Die heutigen Instrumente und Organisationen erfüllen ihren Zweck, sollen aber weiterentwickelt werden. $\mathrm{H}+$ unterstützt seine Mitglieder und fördert gute Ansätze zur Patientensicherheit.

Die Patientensicherheit in den Akutspitälern, Psychiatrie- und Rehabilitationskliniken besteht aus vielen, unterschiedlichen Bausteinen. Dies fängt bereits vor dem Eintritt des Patienten / der Patientin in eine Institution an und geht über den Austrittsprozess hinaus. Für die Spitäler und Kliniken ist es selbstverständlich, bei sämtlichen Arbeitsschritten die Patientensicherheit zu gewähren.

\section{Gesetzliche Grundlagen}

Das Krankenversicherungsgesetz (KVG) legt fest, dass die Spitäler und Kliniken die gesetzlichen und in der Spitalliste definierten Leistungen wirtschaftlich und in der notwendigen Qualität erbringen müssen. Hierfür hat H+ die Branchenlösung Qualität entwickelt. Diese beinhaltet die national einheitliche Vorlage für den Qualitätsbericht und die konsequente Förderung von Qualitätsrichtlinien und Verbesserungsaktivitäten sowie das Informationsportal www.spitalinformation.ch. Weiter ist $\mathrm{H}+$ als Mitträger von Patientensicherheit Schweiz und des Nationalen Vereins für Qualitätsentwicklung in Spitälern und Kliniken (ANQ) ein wichtiger Akteur im nationalen Qualitätsnetzwerk.

\section{Neues nationales Projekt: interprofessionelles Peer Review}

Gemeinsam mit der Verbindung der Schweizer Ärztinnen und Ärzte FMH und der Schweizerischen Vereinigung der Pflegedienstleiter/-innen (SVPL) fördert $\mathrm{H}+$ interprofessionelle Peer Reviews. Das Peer-ReviewPrinzip ist ein bewährtes Mittel, um bei statistischen Auffälligkeiten unter Einbezug von externen Fachkollegen Patientendossiers zu analysieren und - sofern notwendig - Qualitätsverbesserungsmassnahmen zu erarbeiten. Das Peer-Review-Verfahren wird bereits heute in zahlreichen Schweizer Spitälern regelmässig angewandt.

Neu wird ein national einheitliches, interprofessionelles Peer Review vorangetrieben. Gemeinsam mit dem etablierten deutschen Partner Initiative Qualitätsmedizin (IQM) wird dessen international anerkanntes Verfahren nun auf die Schweizer Gegebenheiten ange-

\section{Ergebnisse von Qualitätsmessungen und} Massnahmen zur Patientensicherheit sind auf dem Portal spitalinformation.ch ersichtlich.

passt und in Pilotprojekten erprobt. Dies bedeutet, es wird in drei Landessprachen zur Verfügung gestellt und - als Novum bei IQM - die Pflege in das Verfahren integriert. Dieser interprofessionelle Ansatz erlaubt es, kritische Fälle ganzheitlicher zu betrachten und Qualitätsverbesserungen zur Patientensicherheit gemeinsam zu erarbeiten und umzusetzen.

\section{Qualitäts- und Spitalsuche-Portal spitalinformation.ch}

Dank mehrerer Suchfunktionen ermöglicht das Portal spitalinformation.ch von H+ gezielt ein Spital zu finden, das den individuellen Bedürfnissen entspricht. 
H+ Die Spitäler der Schweiz ist der nationale Verband der öffentlichen und privaten Spitäler, Kliniken und Pflegeinstitutionen. Ihm sind 236 Spitäler, Kliniken und Pflegeinstitutionen als Aktivmitglieder an 369 Standorten sowie knapp 170 Verbände, Behörden, Institutionen, Firmen und Einzelpersonen als Partnerschaftsmitglieder angeschlossen. $\mathrm{H}+$ repräsentiert Gesundheitsinstitutionen mit rund 185000 Erwerbstätigen.

Das Portal unterstützt aber nicht nur Patientinnen und Patienten sowie deren Angehörige bei der Spitalsuche, sondern leistet auch einen entscheidenden Beitrag zur Transparenz der Spital- und Klinikbranche im Qualitätsbereich.

Bei einem bevorstehenden, geplanten Eingriff haben Patienten oft die Wahl zwischen mehreren Spitälern und Kliniken. Für Betroffene oder Angehörige ist es daher nicht immer einfach, sich für einen Anbieter zu entscheiden. Das Portal spitalinformation.ch bietet hierzu Unterstützung. Die Suchfunktionen nach Ort und Leistungsangebot erleichtern die Auswahl und liefern zusätzliche Informationen zur Empfehlung des Haus- oder Spezialarztes. Alle Aktivmitglieder von $\mathrm{H}+$ aus den Bereichen Akutsomatik, Psychiatrie und Rehabilitation sind im Portal aufgeführt. Auch die Suche nach Notfallstationen ist möglich, dabei kann zwischen medizinischem, pädiatrischem, gynäkologischem oder psychischem Notfall differenziert werden.

\section{Transparent, verlässlich und vollständig}

Die Inhalte des Portals beruhen auf validierten Leistungsstatistiken des Bundesamts für Statistik (BFS) sowie den Angaben der Spitäler und Kliniken. Zu den Angaben der Betriebe gehören Qualitäts- und Jahresberichte. Zudem können die Spitäler und Kliniken ihr Profil mit weiteren Daten ergänzen und attraktiv gestalten. Auch Ergebnisse von Qualitätsmessungen und Massnahmen zur Patientensicherheit sind auf der Website pro Betrieb ersichtlich. H+ betreibt das Portal und stellt so die einheitliche Darstellung sicher.

Neben der Website steht auch eine Mobile App «Spitalinformation» für das Smartphone zur Verfügung. Sie basiert auf den gleichen Angaben wie die Website, ist aber hauptsächlich auf Notfälle ausgerichtet.

Korrespondenz: Isabelle Praplan H+ Die Spitäler der Schweiz Lorrainestrasse $4 \mathrm{~A}$ CH-3013 Bern Tel. 0313351111

\section{H+ Branchenlösung}

$\mathrm{H}+$ stellt seinen Mitgliedern eine Vorlage zum Qualitätsbericht zur Verfügung, um der interessierten Öffentlichkeit, den Patienten und Fachpersonen aus dem
Gesundheitswesen einen möglichst gut verständlichen systematischen Überblick über die Qualitätsarbeiten eines Spitals oder einer Klinik zu geben. Die ausgefüllten Qualitätsberichte können die Institutionen auf dem Portal spitalinformation.ch publizieren. 182 Standorte der Spitäler und Kliniken haben 2014 ihren Qualitätsbericht auf www.spitalinformation.ch geladen. Die grosse Mehrheit der Spitäler hat die $\mathrm{H}+$ Vorlage des Qualitätsberichts verwendet.

\section{Patientensicherheit Schweiz}

Die Stiftung für Patientensicherheit ist eine Netzwerkorganisation. Sie analysiert Sicherheitsprobleme und entwickelt, verbreitet und evaluiert Lösungen für Leistungserbringer und fördert den Transfer in die Praxis. All ihre Aktivitäten sollen dazu beitragen, die Patientensicherheit zu verbessern und Fehler in der Gesundheitsversorgung zu vermindern.

Im Bereich Chirurgie hat die Stiftung für Patientensicherheit in Zusammenarbeit mit Experten und Fachorganisationen der invasiv tätigen Berufsgruppen die Grundlagen entwickelt. Zentrales Sicherheitselement ist eine Checkliste, die im Operationssaal angewandt wird. Die Wirksamkeit solcher Checklisten in der Chirurgie wurde in internationalen Studien nachgewiesen. Mit Checklisten kann die Häufigkeit von Todesfällen, Komplikationen und Fehlern reduziert werden. Der Erfolg hängt dabei von der sorgfältigen Einführung und Umsetzung in den Betrieben ab. Das Programm bietet Hilfestellung und Unterstützung. Ziel ist die breite und methodisch richtige Anwendung von Checklisten und damit die Reduktion von Zwischenfällen und Fehlern. Damit wird auch die Sicherheits- und Teamkultur in den Gesundheitsorganisationen gefördert. Das Programm «progress! Sichere Chirurgie» begann im Sommer 2013.

\section{Mehr als 70 medizinische Register}

Ein medizinisches Register kann als systematische Sammlung von populations- oder patientenbezogenen medizinischen und/oder gesundheitsökonomischen Daten verstanden werden. Traditionell sind medizinische Register Instrumente der epidemiologischen Forschung. Immer mehr befassen sich Register jedoch auch mit Qualitätssicherung und Versorgungsforschung, und liefern so einen wesentlichen Beitrag zur Patientensicherheit. Aktuell erfassen schweizweit über 70 medizinische Register Krankheits- und Qualitätsdaten. 